\title{
El concepto de humanidad en Edmund Husserl: delimitación y proyecciones ${ }^{1}$
}

\author{
Edmund Husserl's Concept of Humanity: \\ delimitation and projections
}

Luis Flores Hernández ${ }^{2}$

Pontifícia Universidad Católica de Chile

Recepción: 06 de junio del 2020

Evaluación: 10 de julio del 2020

Aceptación: 20 de julio del 2020

\footnotetext{
Investigación realizada en la Facultad de Filosofía de la Pontificia Universidad Católica de Chile.

2 Doctorat de Troisième Cycle en Philosophie, Mention Logique et Philosophie des sciences, Université d'Aix-en-Provence, France.

Correo electrónico: 1floresh@uc.cl
} 


\title{
Resumen
}

Nuestra propuesta se centra en el concepto de humanidad de Husserl, intentando delimitar la correspondiente familia de conceptos interrelacionados. El foco de nuestro corpus es Die Krisis der europaïschen Wissenschaften und die transzendentale Phänomenologie (Hua VI, 1962) y los artículos para la Revista The Kaizo. Para Husserl, la racionalidad es el concepto clave para interpretar la evolución de la humanidad. El propósito de las investigaciones, que Husserl realiza en estos textos, supone los siguientes aspectos substanciales: 1) Lo que importa es el proceso conducente a una humanidad verdadera. 2) Es esencial a la vida humana el que discurra en la forma del afán. 3) El repertorio conceptual con que Husserl investiga acerca de la humanidad constituye una secuencia ordenada. 4) Lo que es válido para la matemática, en cuanto ciencia pura de la naturaleza, es válido analógicamente para una ciencia pura de la subjetividad. 5) El devenir humano posee unidad y esta es otorgada por la historicidad. 6) El concepto de estilo es aplicado por Husserl al devenir humano. 7) Un concepto importante vinculado al de historicidad es el de Heimatlichkeit. 8) Husserl supone una esencia de la racionalidad, con respecto a la que el racionalismo no es más que un extravío. 9) Distinguimos cuatro intencionalidades en la razón misma. 10) La razón tiene como contraparte negativa a la sinrazón y ciertas opacidades le impiden una transparencia total a la razón.

Palabras clave: humanidad, razón, historicidad, fenomenología, Husserl.

\begin{abstract}
Our proposal is focused on the concept of humanity in Husserl, attempting to delimit the corresponding family of interrelated concepts. The focus of our corpus is Die Krisis der europaïschen Wissenschaften und die transzendentale Phänomenologie (Hua VI, 1962) and the articles in the journal The Kaizo. For Husserl, rationality is the key concept for interpreting humanity's evolution. The purpose of the investigations, that Husserl performs in these texts, supposes the following substantial aspects: 1) What matters is the process leading to a true humanity. 2) It is essential to human life that it goes by in the form of yearning. 3) The conceptual repertory, with which Husserl investigates humanity, constitutes an ordered sequence. 4) What is valid for mathematics, as pure science of nature, is valid analogically for a pure science of subjectivity. 5) Human being becoming possesses unity, which is
\end{abstract}


given by historicity. 6) Husserl applies the concept of style to the human being becoming. 7) A significant concept linked with historicity is that of Heitmatlichkeit. 8) Husserl presupposes an essence of rationality, in which rationalism is nothing more than a deviation. 9) We distinguish four intentionalities concerning reason itself. 10) Reason has unreason as a negative counterpart and certain opacities impede a total transparency of reason.

Keywords: humanity, reason, historicity, phenomenology, Husserl. 
Nuestra propuesta se centra en el concepto de humanidad de Edmund Husserl, intentando delimitar la familia de conceptos interrelacionados -Menschheit, Menschentum, Menschlichkeit und Humanität. Nos interesa determinar su sentido y su interrelación en vistas de sus proyecciones hacia la Antropología, la Ética y la cultura. El foco de nuestro corpus es Die Krisis der europaïschen Wissenschaften und die transzendentale Phänomenologie: Eine Einleitung in die phänomenologische Philosophie (Hua VI, 1962). En ella privilegiamos:

a) La sección I dedicada a "La crisis de las ciencias como expresión de la radical crisis de vida de la humanidad europea", cuyo origen remonta a dos conferencias dictadas en Praga en noviembre de 1935.

b) El § 73. "Conclusión: La filosofía como toma de conciencia de sí mismo, actualización de sí misma de la razón”. Parágrafo incluido por Walter Biemel, el compilador de Hua VI. Dicho texto procede del manuscrito K III 6.

c) Las conferencias de Viena del 7 y 10 de mayo de 1935, incluidas en Hua VI como Tratado (Abhandlung): "Die Krisis des europaïschen Menschentums und die Philosophie".

d) Asimismo, añadimos al foco de nuestro corpus los cinco artículos que Husserl redactó para la revista japonesa The Kaizo entre 1922 y 1923. Tres de ellos aparecidos en 1923 y 1924. Estos ensayos están recogidos en Hua XXVII (1989).

Hay en general en estos textos un movimiento que va desde la mera facticidad descriptible, hacia el seguimiento de la idea (Idee) y del ideal (Ideal). Para Husserl, dicho movimiento está en función de la racionalidad (Rationalität), concepto clave para la interpretación que él hace de la humanidad. En primer lugar, "la razón es lo específico del ser humano como ser que vive en actividades y habitualidades personales" (Hua VI, p. 272; Husserl, 2008, p. $305)^{3}$. En segundo lugar, "decir que la filosofía, ciencia en todas sus formas, es racional es una tautología" (Hua VI, p. 274; Husserl, 2008, p. 306). En tercer lugar, la filosofía "descubre que ésta (la racionalidad) es una idea que se halla en el infinito y en el factum de estar necesariamente en camino (auf dem Wege)" (Hua VI, p. 274; Husserl, 2008, p. 306).

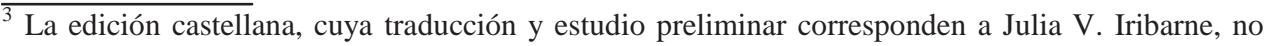
incluye la del Abhandlung antes señalado, cuya versión al castellano es nuestra.
} 
Nos parecen substanciales en Husserl los siguientes aspectos:

1. No es el terminus ad quo o el terminus ad quem lo que importa, sino el proceso que conduce a una humanidad más verdadera, más auténtica, "el proceso de llegar a ser hombres" (Menschenwerdens) (Hua XXVII, p. 55; Husserl, 2002, p. 60). Por eso, es posible que el término „Humanismus” no sea usado por Husserl, pues implicaría un congelamiento del devenir. Incluso la ciencia estricta, ligada a ese proceso, "no es un ente objetivo, sino el proceso de devenir (werden) de una objetividad ideal" (Hua XXVII, p. 55; Husserl, 2002, p. 60). Por eso, todo se juega en este devenir y eso justifica el aserto husserliano: "todo esto no ha de entenderse en sentido estático, sino dinámico-genético" (Hua XXVII, p. 55; Husserl, 2002, p. 60). Pero este devenir es orientado y, por eso, es desarrollo (Entwicklung), "en el sentido de un continuo ir escalonando grados de valor (fortgesetzter Wertstufung)" (Hua XXVII, p. 51; Husserl, 2002, p. 56). Se trata, respecto de una colectividad (Gemeinschaft), de "ponerse en el camino (auf die Bahn) de su desarrollo progresivo" (Hua XXVII, p. 55; Husserl, 2002, p. 60). Y ello importa porque el desarrollo en sí mismo no cualifica el sentido del cambio. Husserl introduce dos formas de desarrollo ético, lo que supone distinguir entre la dirección y el sentido. Uno con valor positivo, como elevación (Erhöhung) y otro con valor negativo, como decadencia (Herabsinken). El desarrollo supone estadios (Stufen) y fases que permiten la conexión con el valor absoluto: "Una vez que la ciencia ha conquistado el estadio de la ciencia auténtica, del verdadero logos, su devenir se convierte en un sistema procesual de valores absolutos (ein Werdenssystem absoluter Werte) y de un valor absoluto ya realizado en cada estadio" (Hua XXVII, p. 55; Husserl, 2002, pp. 60-61). Así, el devenir de la ciencia auténtica tiene un "horizonte de futuros incrementos y elevaciones de valor (Werterhebungen)" (Hua XXVII, p. 55; Husserl, 2002, p. 61). En cuanto a la colectividad que se construye a sí misma y hace suya la idea que le es propia, en cada estadio, "tiene aún ante sí horizontes abiertos, indeterminaciones pendientes de determinarse" (Hua XXVII, p. 56; Husserl, 2002, p. 61).

2. El proceso de lo que podríamos llamar "humanización" es aquel en que comenzamos a ser lo que somos - como diría Píndaro, sé el que eres-, en que lo humano ya no nos es extraño -al decir de Terencio, "soy hombre; nada de lo que es humano lo considero ajeno a mí" (Herrero, 2007, p. 120)-, pues se nos hace entraño, y, por ende, entrañable. Dicha humanización no 
es entonces la aplicación rutinaria y automática de reglas éticas, sino que surge "en un constante combate" (Kampf) (Hua XXVII, p. 58; Husserl, 2002, p. 63), "en una lucha diaria" (Hua XXVII, p. 45; Husserl, 2002, p. 48). No hay aquí sólo un imperativo categórico operando, hay una materialidad de lucha (Kampf), de fe (Glaube), de empuje o brío (Schwung), de afán (Streben), de fuerza de pulsión o de impulso o propulsora (Triebkraft). Así Husserl dice: "De la esencia de la vida humana es, además, el que discurra constantemente en la forma del empeño, del afán (des Strebens)" (Hua XXVII, p. 25; Husserl, 2002, p. 26). La humanización se sitúa entonces en el escenario de los motivos y de las motivaciones, allende las causas y los efectos: "para nosotros resulta más importante comprender el camino de la motivación, el de la donación de sentido y el de la creación de sentido, que conduce de la mera reorientación, es decir, del mero

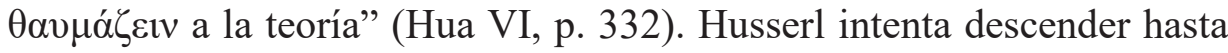
los estratos más originarios desde donde surgen nociones como protomotivo, protofenómeno, protosuelo, protométodo.

3. El repertorio de términos usados por Husserl para hablar de humanidad constituye una secuencia ordenada:

En primer lugar, en el estrato descriptivo más básico aparece die Menscheit, humanidad en cuanto inserta en un mundo circundante (Umwelt) y una cultura, la que es definida como "el conjunto total de logros (Leistungen) que vienen a la realidad merced a las actividades (Tätigkeiten) incesantes de los hombres en sociedad y que tienen una existencia (Dasein) espiritual duradera en la unidad de la conciencia colectiva y de la tradición que la conserva y prolonga" (Hua XXVII, p. 21; Husserl, 2002, p. 22). La distinción husserliana entre Tätigkeit y Leistung corresponde, al menos en cierta medida, a la distinción aristotélica entre energeia y ergon. De hecho, Wilhem von Humboldt aplicó esta distinción al lenguaje, distinguiendo entre Sprache como energeia y como ergon (von Humboldt, 2003). A su vez, „,Menscheit” tiene una aproximación metafórica y otra metafórico-conceptual:

a) La primera se caracteriza así: "Es como un mar en el que los seres humanos y los pueblos son las olas que se forman fugazmente, que cambian y de nuevo desaparecen, unas onduladas más rica y complejamente, otras más primitivamente" (Hua VI, p. 319). Se trata de una totalidad fluyente, semejante al río heraclitano de la conciencia, del 
que Husserl nos habla, por ejemplo, en Die Idee der Phänomenologie: "Nos movemos en el campo de los fenómenos puros. ¿Sin embargo, por qué digo campo? Es, más bien, un eterno río heraclitano de la conciencia" (Hua II, p. 47; Husserl, 1982, p. 59).

b) La segunda es definida así: "En tal proceder, aparece la humanidad (Menscheit) como una única vida de hombres y pueblos, unida por sólo relaciones (Bezüge) espirituales con una abundancia de tipos de humanidad y cultura, sin embargo, fluyendo las corrientes unas en otras" (Hua VI, p. 319). Lo importante es que la noción empírica de tipo corresponde a la Menschheit.

En segundo lugar, aparece das Menschentum, que es humanidad aún en un sentido descriptivo, pero más abstracto, que posee modos de existencia (humanidad antigua, medieval, renacentista o moderna), que plantea cuestiones y problemas específicos, que necesita ser adjetivada. Posee una definición conceptual: "Humanidad (Menschentum) en general es, esencialmente, ser humano en humanidades (Menschheiten) vinculadas generativa y socialmente" (Hua VI, p. 13; Husserl, 2008, p. 59).

En tercer lugar, en las conferencias de Viena, Husserl introduce los términos de die Menschlichkeit (Hua VI, p. 338) y das Menschliche (Hua VI, p. 341) para apuntar a lo más específico del ser humano (in seiner spezifischen Menschlichkeit) (Hua VI, p. 341): el hombre como persona, el hombre en su razón (Hua VI, p. 338). En este mismo sentido específico, Husserl señala: "así como el ser humano, incluso el papúa, representa, frente al animal mismo un nuevo estadio de animalidad, así la razón filosófica representa un nuevo estadio en la humanidad (Menschlichkeit) y su razón" (Hua VI, pp. 337-338). Más aun, en el siguiente texto, contrasta Menschlichkeit con Menschentum: "La humanidad (Menschentum) de la superior humanidad (Menschlichkeit) o razón exige, por consiguiente, una filosofía auténtica" (Hua VI, p. 338).

En cuarto lugar, ya en el ensayo Kaizo I, aparece el término die Humanität, para ser entendido como idea, en contraste con die Menschheit. Esto supone el alejamiento de lo fáctico en dirección a la idealidad; es el salto al limes que nos otorga el infinito. Ahora bien, Husserl no confunde, ya desde las Logische Untersuchungen, entre la idea (Idee) y el ideal o arquetipo (Urbild). La primera responde a una abstracción que implica el paso de lo múltiple a lo uno, en el sentido de una racionalidad explicativa. 
El segundo responde a una racionalidad normativa, la del deber ser. En suma, aquí "humanidad" puede ser una idea, pero también un ideal. Ahora bien, tres notas son esenciales a Humanität: la primera es la reflexión, la que se vincula con autorreflexión (Selbstbesinnung), autocomprensión (Selbstverständnis), autodesvelamiento (Selbstenthüllung). La segunda es la responsabilidad (Verantwortung). ¿De qué responsabilidad se trata? De una doble responsabilidad: "La responsabilidad totalmente personal por nuestro ser propio y verdadero como filósofos, en nuestra íntima vocación personal, comporta en sí, al mismo tiempo, la responsabilidad por el verdadero ser de la humanidad (Menschheit)" (Hua VI, p. 15; Husserl, 2008, pp. 60-61). La segunda nota conviene más con el ideal. Así se refiere como ideal de configuración de "una humanidad auténticamente humana (eine humane Menschheit)" al criterio que otorga la humanitas (Humanität) ${ }^{4}$, en cuanto se trata "de la humanidad (Menschheit) en que la razón se ha cultivado y objetivado en la figura del logos" (Hua XXVII, p. 55; Husserl, 2002 , p. 60). La tercera nota es la libertad presente en el movimiento de libertad religiosa (Hua XXVII, p. 63; 2002, p. 69), en la figura del movimiento de libertad filosófica (Hua XXVII, p. 73; Husserl, 2002, p. 80), en "la peculiaridad del movimiento de liberación (Befreiung) que se inicia en la nación griega y en ella se consuma en un trecho fundamental como creación de una nueva forma cultural: la filosofía" (Hua XXVII, p. 73; Husserl, 2002, p. 80).

4 La argumentación central de Husserl es analógica. Lo que es válido para la matemática, en cuanto ciencia pura de la naturaleza, es válido analógicamente para una ciencia pura de la subjetividad, la fenomenología trascendental o lo que podríamos llamar una neumatología; la ciencia del espíritu. La analogía se basa en el paso al límite que permite alcanzar la idealidad. La analítica del espíritu es perfilada así: "La formación de un método real (wirklich) para aprehender la esencia fundamental del espíritu en sus intencionalidades y construir, a partir de ahí hasta el infinito, una consecuente analítica del espíritu, condujo a la fenomenología trascendental" (Hua VI, p. 346). Como Husserl dice en Kaizo I: "Falta aquí, justamente, la ciencia apriórica paralela, la mathesis del espíritu y de la condición humana (Humanität) por así decir" (Hua XXVII, p. 7; Husserl, 2002, p. 5). Lo que no implica una geometría de las vivencias -como

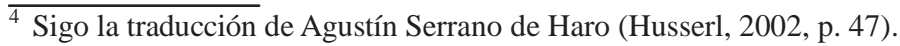


plantea en Ideen I: "y por eso preguntamos especialmente si una fenomenología como una 'geometría' de las vivencias tenga que ser constituida o pueda ser constituida” (Hua III, p. 165), pues en la fenomenología trascendental los conceptos son morfológicos y no exactos.

A su vez, Husserl define la ética pura así: "es la ciencia de la esencia y formas posibles de una vida en renovación tomada en generalidad pura (apriórica)" (Hua XXVII, p. 20; Husserl, 2002, p. 21). Distingue la ética pura de la empírica-humana y divide la ética en ética individual y social. El asunto es que analógicamente lo que es válido para la ética individual lo es nuevamente de un modo analógico para la ética social. Sin embargo, la comunidad de hombres de bien no ha de ser entendida como la simple sumatoria o agregación de individuos buenos.

5. El devenir humano (Menschenwerden) no es caótico, posee unidad. Ésta es otorgada por la historicidad (Geschichtlichkeit), en el sentido más general, la que es definida como "historicidad (Historizität) generativa originaria” (Hua VI, Beilage XXVI, p. 502) y que es un universal que pertenece a la existencia (Dasein) humana, que es un devenir unitario (ein einheitliches Werden) según las personas, en las personas. Y la historicidad, como un medio ambiente (Umwelt) humano, "en la multiplicidad de sus figuras, puede ser considerada como unidad de un "organismo"” (Hua VI, Beilage XXVI, p. 502). La historicidad según Husserl es diferente a la de Heidegger: "Historicity, for Husserl, does not have quite the same technical sense that it has in Heidegger (...) Note here that Husserl characterizes historicity as a universal and necessary property belonging to human existence. As we shall see below, Heidegger makes historicity into an existential characteristic of Dasein" (Moran, 2012, p. 155).

6. Nos parece importante el concepto operativo de estilo, aplicado al dominio existencial del devenir humano en la actitud natural: "Este movimiento [el de una reformación (Neubildung) progresiva] transcurre desde un principio comunicativamente; despierta un nuevo estilo de existencia personal (einen neuen Stil personalem Daseins) en su círculo vital, en el comprender reviviendo [Nachverstehen $]^{5}$ de un devenir (Werden) correspondiente nuevo" (Hua VI, p. 322). Asimismo, la humanidad (Menschheit) vive en una u otra actitud. ¿Y qué es una actitud (Einstellung)? "Una actitud

\footnotetext{
$\overline{5}$ Sigo la traducción hecha de este término por Antonio Zirión (Husserl, 1997, p. 504).
} 
significa, en términos generales, un estilo (Stil) habitualmente fijo de la vida de la voluntad en direcciones de voluntad o en intereses delineados merced a ello en fines últimos, en rendimientos culturales, cuyo estilo global (gesamter Stil) es, por consiguiente, con ello determinado" (Hua VI, p. 326). En suma, "en este estilo permanente como forma normal transcurre la vida cada vez determinada" (Hua VI, p. 326).

Ahora bien, un cambio de actitud o mutación de la misma (Umstellung, Wendung, Umwendung, Umwandlung, Umbildung) implica un cambio de estilo. Husserl no entiende estas mutaciones como meros cambios o cambios infinitesimales: "Pudiera parecerme que yo, el pretendido reaccionario, soy mucho más radical y revolucionario (revolutionär) que los que hoy en día se manifiestan en palabras tan radicalmente" (Hua VI, p. 337). Se trata, más aun, de una revolucionización (Revolutionierung) (Hua VI, p. 325) de la historicidad. De esta manera el término "Revolution" parece más aplicable al dominio de los eventos. En cambio, la revolucionización implica un cambio en el dominio categorial, constituye un estrato metarrevolucionario.

7. ¿Cuál es el punto de quiebre de Husserl para fundamentar su apuesta por un racionalismo no ingenuo? El ser humano es un animal que, según el

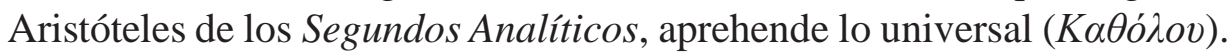
Husserl señala al respecto: "El ser humano de la vida cotidiana, sin embargo, no carece de razón, él es un ser pensante, él tiene el kathólou frente al animal, por eso él tiene habla, descripción, infiere, formula preguntas acerca de la verdad, [él comprueba, argumenta y se decide racionalmente]"6 (Hua VI, p. 270; Husserl, 2008, p. 303). Entonces humanización supone al menos racionalización, y ésta, al menos, universalización, mediante el acceso a las ideas. Estas son formaciones de sentido (Sinngebilde), "que implican infinitudes intencionales" (Hua VI, p. 322). Sin embargo, la universalización husserliana no corresponde a la globalización, hecha de acuerdo a ciertos patrones económico-tecnológicos. En Husserl, la humanización pasa por la persona y ésta supone la unicidad. Así, los conceptos husserlianos de estilo y de historicidad pueden permitir equilibrar un proceso de creciente homogeneización mundial. El estilo permite la diversidad cultural, en cuanto es un concepto existencial que se aplica a la

${ }^{6}$ El pasaje entre paréntesis rectos no está en la traducción. 
existencia personal. A su vez, el nuevo tipo de historicidad, que surge con un cierto tipo de desarrollo, cobra un perfil propio, pues "se destaca de la historia general" (Hua VI, p. 323). Por otra parte, un concepto vinculado al de historicidad es el de Heimatlichkeit. Se trata de un "parentesco especial, íntimo en el espíritu, que pasa por ellas [las naciones europeas]" (Hua VI, p. 320). Este parentesco implica diferencias, pero a la vez identifica. Es a la vez unicidad y unidad: "Es así algo como una fraternalidad (Geschwisterlichkeit) que en este círculo nos da la conciencia de una terruñidad (Heimatlichkeit)" (Hua VI, p. 320). Se trata de una "unidad de parentesco familiar" (Hua VI, p. 320). Dicha unidad depende de si es considerada ad intra o ad extra. En el primer caso, los miembros de dicho círculo se viven "sólo mutuamente como coterráneos (Heimgenossen)" (Hua VI, p. 320). En el segundo, los miembros externos nos experimentan (erleben) como extranjeros. En suma, la diferencia esencial entre terruñidad (Heimatlichkeit) y extrañeza (Fremdheit) constituye una categoría fundamental de la historicidad. Así los griegos llamaban "ßó $\rho \beta \alpha \rho \varsigma$ ”, término usado como onomatopeya de un lenguaje ininteligible, al no griego. Por otra parte, ¿es posible superar la Heimatlichkeit? Merleau-Ponty cita un pasaje de Les Sept Piliers de la Sagesse de Thomas Edward Lawrence (Lawrence, 1936, p. 43), en donde éste al final de un proceso deliberado de arabización, se quiebra y reconoce su fracaso. Merleau-Ponty comenta que: "Para asimilar completamente una lengua, sería necesario asumir el mundo que ella expresa y no se pertenece jamás a dos mundos a la vez" (Merleau-Ponty, 1945, pp. 218-219). Es cierto que, para Husserl, el telos espiritual de la humanidad europea yace en el infinito, a diferencia de otras humanidades, aunque conlleve los telos singulares de las naciones y de los hombres ${ }^{7}$.

8. Respecto de la racionalidad, Husserl piensa que el racionalismo no es más que una figura evolutiva de la ratio. Es un extravío, aunque comprensible. ¿En qué consiste el extravío? No es la esencia de la Rationalität la que está en juego. Su extravío consiste "solamente en su enajenabilidad (Veräußerlichung), en su envolverse con los capullos (Versponnenheit) del 'naturalismo' o del 'objetivismo"” (Hua VI, p. 347). A propósito del

\footnotetext{
La humanidad europea es una idea-fin (Zweck-Idee), por ende no se identifica con un determinado factum histórico, social, genético, geográfico, etc. Este factum varía temporalmente y está sujeto a aproximaciones como a desviaciones con respecto a la idea-fin.
} 
racionalismo extraviado, Husserl nos advierte que: "Esto no da derecho a la opinión de que la racionalidad como tal sea perjudicial o que sea sólo de significación subordinada en el todo de la existencia humana" (Hua VI, p. 337). En suma, no se trata del retorno a la Aufklärerei ${ }^{8}$, a "un intelectualismo que se pierde en una teoría ajena al mundo" (Hua VI, p. 337). Por lo demás, el racionalismo del siglo XVIII era una ingenuidad, cuyas últimas implicaciones conducen a un contrasentido.

La alternativa al racionalismo ingenuo es el irracionalismo "tan celebrado que se nos propone" (Hua VI, p. 14; Husserl, 2008, p. 59). Husserl señala también su contrasentido, porque el irracionalista elabora un discurso y solicita audiencia. Entonces Husserl muestra su contradicción performativa, pues su acto comunicativo de intentar convencernos presupone la racionalidad que niega: “¿No debe él [el irracionalismo] convencernos, si hemos de prestarle atención, acerca de cómo examina y funda racionalmente?" (Hua VI, p. 14; Husserl, 2008, p. 59). Husserl se pregunta si esta irracionalidad no es una mala racionalidad, una racionalidad corta de miras, "peor que aquella del viejo racionalismo" (Hua VI, p. 14; Husserl, 2008, pp. 59-60). En suma, se trata de una razón perezosa: “¿No es hasta la de la 'razón perezosa' que rehúye la lucha a favor de la claridad de lo pre-dado último y la de los fines y los caminos verdadera y racionalmente predelineados?" (Hua VI, p. 14; Husserl, 2008, p. 60). Husserl subsumiría, bajo este concepto, desde el positivismo -"El positivismo, por así decir, decapita la filosofía" (Hua VI, p. 7; Husserl, 2008, p. 53)- hasta, pudiera ser, el pensiero debole de Gianni Vattimo (Vattimo y Rovatti, 1983).

Husserl es una solitaria voz que clama en el desierto. Es diciembre de 1935, fecha de las conferencias de Praga, y los nubarrones del nazismo despuntan en el cielo de Europa. Por este motivo, la renovación que lo ocupa desde los artículos de Kaizo conforme al concepto de Erneuerung y, posteriormente, en las conferencias de Praga, según el concepto de reformación (Neubildung), no puede surgir al alero del mero academicismo universitario. Husserl se pregunta: “¿Queríamos nosotros escuchar aquí sólo un discurso académico?" (Hua VI, p. 15). Ciertamente, la filosofía como mera erudición histórica, como seguro de vida formateado según

\footnotetext{
${ }^{8}$ Expresión peyorativa para designar a la Aufklärung, "tradicional desde Hegel” (Husserl, 1976, p.
} 371). 
los cánones de una carrera académica -el publish or perish, los rankings universitarios, la indexación de revistas académicas, etc.- no basta, diría Husserl, dadas la crisis y la indigencia. Por eso nos dice: "Precisamente en esto se halla nuestro propio desamparo (Not), el de todos nosotros, quienes no hacemos filosofía literaria (Literaten-Philosophen) sino que, formados por los auténticos filósofos del gran pasado, somos y queremos ser viviendo de la verdad, y sólo viviendo en nuestra propia verdad" (Hua VI, p. 15; Husserl, 2008, p. 60).

Tampoco la salida es posible contentándose con una racionalidad unilateral, pues ésta "puede en efecto convertirse en un mal" (Hua VI, p. 338). Mientras esta unilateralidad sea provisoria no atenta a la esencia de la razón. Pero ésta es esencialmente sistémica. A la razón analítica hay que contrapesar la sintética: "Ninguna línea de conocimiento, ninguna verdad particular, tiene el derecho a ser aislada y absolutizada" (Hua VI, p. 339). Por eso, se requiere una razón vigilante: "Ahí amenaza constantemente el caer en unilateralidades y satisfacciones precipitadas, que se vengan en subsecuentes contradicciones" (Hua VI, p. 338). Una de las exigencias del desarrollo científico y filosófico es la especialización. Thomas S. Kuhn ve en ésta un rasgo esencial a la ciencia normal (Kuhn, 1970). Y la filosofía misma se especializa, pero Husserl nos advierte: "A ello se agrega la necesidad y, sin embargo, de nuevo, la peligrosidad de la especialización" (Hua VI, p. 338). Por otra parte, el racionalismo de Husserl se basa en dos axiomas. Según el primero, las ideas filosóficas superan a los poderes empíricos: "Y, sin embargo, las ideas son más fuertes que todos los poderes empíricos" (Hua VI, p. 335). Según el segundo, lo ideal se contrapone a lo real en la ciencia y en la filosofía como formaciones culturales: "Con una palabra, lo que la conducta científica logra, no es real, sino ideal" (Hua VI, p. 323).

9. Allende Husserl, distinguimos cuatro intencionalidades de la razón:

a) La cognitiva, orientada a la producción del conocimiento y guiada por el criterio de verdad como adequatio o correspondencia.

b) La deóntica, orientada a la producción de ideales o de Zweck-Ideen. Es la función arcóntica de la filosofía y, por ende, de la razón: "La filosofía ha de ejercer constantemente, en una humanidad europea, su función como la función arcóntica de toda la humanidad” (Hua VI, p. 336). 
c) La sintomática o expresiva, en virtud de la que la razón posee estratos latentes y patentes. Husserl nos habla del "movimiento infinito de la razón latente a la manifiesta" (Hua VI, p. 13; Husserl, 2008, pp. 58-59). Se trata entonces de la fenomenología como arqueología, que opera sobre lo oculto, lo oscuro y lo latente para que devenga lo manifiesto, lo claro y lo patente. La verdad no ha de ser entendida aquí como adequatio rei et intellectus, sino como alétheia, como manifestación del logos, como logofanía.

d) La estética, por la que la razón es intencionada arquitectónicamente, es tematizada en la belleza de su despliegue lúdico, de su simetría, de su armonía, etc. Husserl sólo se refiere a las normas tradicionales de la belleza a propósito de la idea general de la verdad en sí (Hua VI, p. 334).

10. La contraparte negativa de la razón es la sinrazón (Unvernunft), distinción en curso desde Ideen I: "Naturalmente, es también de considerar en todas partes la 'sinrazón' como contraparte negativa de la razón” (Hua III, p. 353). El dominio de la filosofía pareciera ser sólo el discurso de la razón. Sin embargo, Husserl abre horizontes que no son racionales o que pertenecen a una racionalidad más blanda; en todo caso, a opacidades de la razón. Examinemos algunas de estas:

a) El papel que juega, por ejemplo, die Vorahnung: "Todo esto no quiere ser una interpretación especulativa de nuestra historicidad, sino expresión de un presentimiento (Vorahnung) vivo que surge en una reflexión desprejuiciada" (Hua VI, p. 321). Sin embargo, el presentimiento trasciende el barrunto, pues nos conduce a una certeza: "Éste nos da una conducción intencional para ver conexiones (Zusammenhänge) sumamente significativas, en cuya persecución lo presentido se convierte en certeza profunda" (Hua VI, p. 321). Y así Husserl concluye que: "El presentimiento es el indicador de camino, al modo de los sentimientos (gefühlsmäßiger Wegweiser), de todos los descubrimientos" (Hua VI, p. 321) ¿Es la concesión a l'esprit de finesse de Pascal, a esas razones del corazón que la razón no conoce? Dice Pascal: "El corazón (coeur) tiene sus razones que la razón no conoce; se lo sabe en mil cosas" (Pascal, 1964, p. 146). En todo caso, el presentimiento pertenece al contexto de descubrimiento (Reichenbach, 1938), o al ars inveniendi de Leibniz. 
b) Respecto de la ciencia, Husserl dice sorprendentemente que ésta incluye instinto: "Descubrimiento es una mezcla de instinto (Instinkt) y método" (Hua VI, p. 39; Husserl, 2008, p. 83). Con todo, se pregunta si acaso esto es, en sentido estricto, ciencia. El concepto de instinto científico en Husserl cumple un papel análogo al concepto de sagacidad científica de William Whewell: "The Conceptions by which Facts are bound together, are suggested by the sagacity of discoverers. This sagacity cannot be taught (Whewell, 1847, p. 467). Este sitúa el origen de las ciencias empíricas en la sagacity. Ahora bien, este término deriva del latín sagacitas, que significa finura de olfato en los perros. Por lo tanto, en la mesa del banquete filosófico, en un extremo de la misma, René Descartes, autor del Discours de la Méthode y de las Regulaead directionem ingenii (Descartes,1953) frunce el ceño ante la tesis husserliana; en el otro extremo, Paul K. Feyerabend, autor de Against Method (Feyerabend, 2010), sonríe con aprobación. En suma, ¿qué son entonces las físicas de Galileo, Newton y Einstein? ¿Predomina en ellas el instinto o el método?

c) Ante la crisis de la existencia europea, la salida positiva constituye una épica de la razón: “el renacimiento de Europa a partir del espíritu de la filosofía mediante el heroísmo (Heroismus) de la razón que vence definitivamente el naturalismo" (Hua VI, pp. 347-348). No se trata precisamente de una reengineering de la humanidad, pero entonces ¿qué es? Es la filosofía como epopeya de la razón.

d) El proceso de la racionalización de la cultura europea propuesto por Husserl se destaca como una figura sobre un trasfondo bíblico, enfrentando "el mayor peligro de sumergirnos en el diluvio escéptico ( $i n$ der skeptischen Sintflut) y con ello renunciar a nuestra propia verdad" (Hua VI, p. 12; Husserl, 2008, p. 58). ¿Qué es entonces esta alegoría bíblica que presupone que la fenomenología trascendental es el Arca de Noé en el mar de la humanidad y que es amenazada por el diluvio escéptico? 


\section{Referencias}

Descartes, R. (1953). Oeuvres et Lettres. Paris: Gallimard.

Feyerabend, P. (2010). Against Method. London/New York: Verso.

Herrero, V. (2007). Verbi gratia. Diccionario de expresiones latinas. Madrid: Gredos.

Husserl, E. (1950). Ideen zu einer reinen Phänomenologie und Phänomenologische Philosophie. (Hua III). Erstes Buch: Allgemeine Einführung in die reine Phänomenologie. Biemel, W (Hrsg.). Den Haag: Martinus Nijhoff.

Husserl, E. (1958). Die Idee der Phänomenologie. Fünf Vorlesungen (Hua II). Biemel, W (Hrsg.). Den Haag: Martinus Nijhoff.

Husserl, E. (1962). Die Krisis der europaïschen Wissenschaften und die transzendentale Phänomenologie: Eine Einleitung in die phänomenologische Philosophie (Hua VI). Biemel, W (Hrsg.). Den Haag: Martinus Nijhoff.

Husserl, E. (1970). The Crisis of European Sciences and Transcendental Phenomenology. An introduction to Phenomenological Philosophy. (Trad. Carr, D). Evanston: Northwestern University Press.

Husserl, E. (1976). La crise des sciences européeennes et la phénoménologie Transcendantale. (Trad. Granel, G). Paris: Gallimard.

Husserl, E. (1982). La idea de la fenomenología. Cinco lecciones. (Trad. García-Baró, M). México: Fondo de Cultura Económica.

Husserl, E. (1989). Aufsätze und Vorträge (1922-1937) (Hua XXVII). Sepp, R (Hrsg.). Dordrecht: Kluwer.

Husserl, E. (1997). Ideas relativas a una fenomenología pura y una filosofía fenomenológica. (Trad. Zirión, A). Libro Segundo: Investigaciones fenomenológicas sobre la constitución. México: Universidad Nacional Autónoma de México.

Husserl, E. (2002). Renovación del hombre y de la cultura. Cinco ensayos.

(Trad. Serrano de Haro, A). Barcelona: Anthropos. 
Husserl, E. (2008). La crisis de las ciencias europeas y la fenomenología trascendental. (Trad. Iribarne, J). Buenos Aires: Prometeo Libros.

Kuhn, T. S. (1970). The Structure of Scientific Revolutions. Second Edition, enlarged. Chicago: The University of Chicago Press.

Lawrence. T. E. (1936). Les Sept Piliers de la Sagesse. Paris: Payot.

Merleau-Ponty, M. (1945). Phénoménologie de la perception. Paris: Gallimard.

Moran, D. (2012). Husserl's Crisis of the European Sciences and Transcendental Phenomenology. An Introduction. Cambridge: Cambridge University Press.

Pascal, B. (1964). Pensées. Paris: Garnier Frères.

Reichenbach, H. (1938). Experience and Prediction. An Analysis of the Foundations and the Structure of Knowledge. Chicago: University of Chicago Press.

Torretti, R. (1999). The Philosophy of Physics. Cambridge: Cambridge University Press.

Vattimo, G. e Rovatti, P. A. (1983). Il pensiero debole. Milan: Feltrinelli.

Von Humboldt, A. (2003). Über die Verschiedenheit des menschlichen Sprachbaues: Über die Sprache. Wiesbaden: Fourier.

Von Humboldt, W. (2003). Über die Verschiedenheit des menschlichen Sprachbaues und ihren Einfluss auf die geistige Entwicklung des Meschengeschlechts: Über die Sprache. Wiesbaden: Fourier.

Whewell, W. (1847). The Philosophy of the Inductive Sciences, founded upon their History. London: J. W. Parker. 